\title{
Preliminary Simulations of LFI Main Beam Using measured Feed Horn Patterns
}

\author{
Paola Platania* \\ IFP-CNR \\ Via Cozzi 53, 20123, Milan, Italy \\ E-mail: platania@ifp.cnr.it \\ Ocleto D'Arcangelo, Lorenzo Figini, Carlo Sozzi \\ IFP-CNR \\ Via Cozzi 53, 20123, Milan, Italy \\ E-mail: ocleto@ifp.cnr.it, figini@ifp.cnr.it, sozzi@ifp.cnr.it
}

Maura Sandri, Fabrizio Villa

IASF-INAF

Via Gobetti 101, 40129, Bologna, Italy

E-mail: sandri@iasfbo.inaf.it, villaliasfbo.inaf.it

\begin{abstract}
We present the ongoing activity dedicated to the simulation of Planck LFI main beam at 30 and $70 \mathrm{GHz}$ using the Flight Model (FM) measured Feed Horn patterns as input. In this context, apart from measurements performed during the feed horn qualification test campaign, dedicated measurements have been performed with the aim to i) investigate the optical systematic induced by real horns and ii) to identify the best LFI horns model for flight pattern predictions. Preliminary comparisons of the main beam are reported with the case in which the simulated feed horn are used. Some characteristic parameters are shown for each simulated main beam.
\end{abstract}

$C M B$ and Physics of the Early Universe

20-22 April 2006

Ischia, Italy

${ }^{*}$ Speaker. 


\section{Introduction}

The LFI Flight Model (FM) feed horn measurements have been performed at the Laboratorio di Microonde of the Istituto di Fisica dei Plasmi (CNR) in Milano, with an ABmm 8-350-4 Vector Network Analyser in an anechoic chamber. The LFI feed horns were mounted on a rotational stage, directly driven by the VNA with a precision of $0.01^{\circ}$ : amplitude and phase patterns were acquired between $\pm 90^{\circ}$ at five frequencies, the central one (30, 44 and $\left.70 \mathrm{GHz}\right)$ and at $\pm 5 \%$ and $\pm 10 \%$. Standard rectangular $(44$ and $70 \mathrm{GHz})$ and conical $(30 \mathrm{GHz})$ horns were used as receivers. The co-polar component was measured in the two principal planes $(\mathrm{E}, \mathrm{H})$, while also the cross-polar component is detected in the $\pm 45^{\circ}$ polarisation planes where it reaches its maximum value [1]. Even if it was possible to detect a cross-polar level of about -30/-40dB, the cross-polar component of the receiving standard horns is a limiting factor and it hides the real behaviour of the LFI feed horn. Thus, for one $70 \mathrm{GHz}$ feed, also an LFI feed horn was used as a receiver, to determine the impact of the non ideal knowledge of the cross-polar component in the simulation. Being the crosspolar pattern difficult to measure due to the low signal to noise ratio, it is very useful to assess the reliability of the simulation in that condition.

Main beam simulations have been carried out for two feed horns at 30 and $70 \mathrm{GHz}$ considering the feed as source and computing the pattern scattered by the two reflectors of the Planck telescope using the software GRASP9, an integrated tool for general reflector analysis developed by TICRA, using the Physical Optics as the analysis method [2]. The results are shown as pattern cuts in the $\mathrm{E} / \mathrm{H} / \pm 45^{\circ}$ planes for the co- and cross-polar components according to the Ludwig 3rd definition [3]. Main beams have also been computed in the UV spherical polar grids, in which $u=\sin \theta_{b f} \cos \phi_{b f}$ and $v=\sin \theta_{b f} \sin \phi_{b f}$ where bf means beamframe (each main beam has been computed is its own coordinate system) [4], and $\theta$ and $\phi$ follow GRASP9 angle definition. Some characteristic parameters are shown for each simulated main beam, to compare cases in which simulated or measured feed horns are used as input.

\section{2. $30 \mathrm{GHz}$ feed horns and main beam simulations}

The feed considered in this work is the FM n.40101. The pattern measurements have been carried out for the $\mathrm{E} / \mathrm{H} / \pm 45^{\circ}$ planes; cross-polar component has been measured only at $\pm 45^{\circ}$ and set to zero along the two principal planes. The receiver feed is a standard conical horn with a directivity of nearly $21 \mathrm{dBi}$.

The simulated feed horn pattern has been produced with Mode Matching (MM) techniques [5] combined with a Finite Element Method (FEM) along 72 planes (at the INAF - Osservatorio Astrofisico di Arcetri by R.Nesti) (see Fig.1). The cross-polar components in the E/H planes are at $\sim 70 \mathrm{~dB}$ lower than the level of the $\pm 45^{\circ}$ planes, that are comparable with the measured ones, even if different in the detailed shape.

For the main beam simulation at $30 \mathrm{GHz}$ the location and orientation of the feed \#27 has been chosen. Location and orientation are defined with respect to Reference Detector Plane and according to GRASP9 angle definition [4]. The GRASP9 simulation of the main beam shows a good agreement between the two cases, reflected by the beam characteristics reported in Table 1 . 

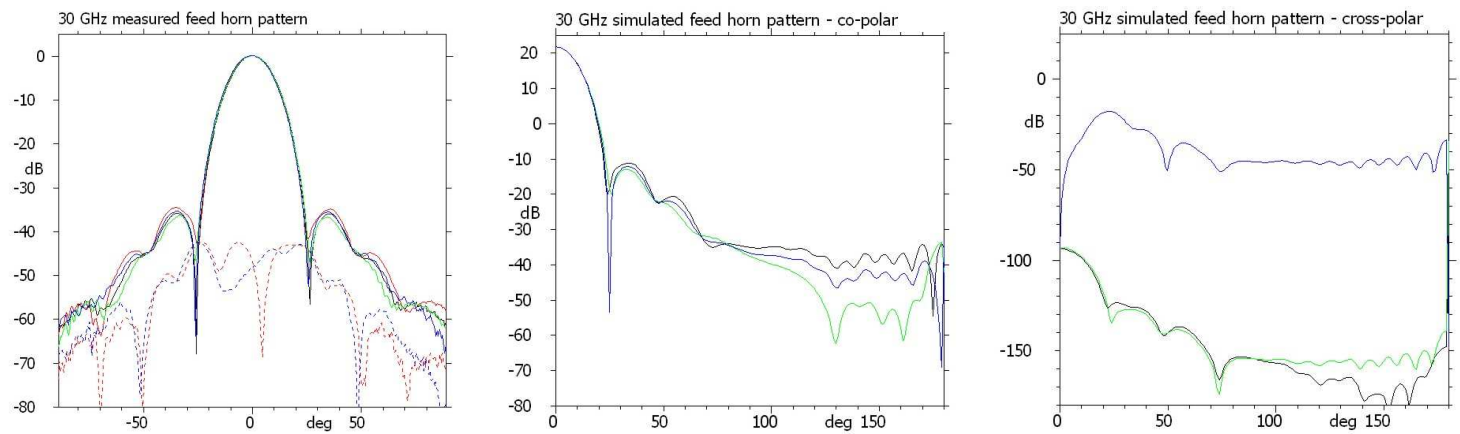

Figure 1: Left: $30 \mathrm{GHz}$ measured feed horn pattern, co- (solid) and cross-polar (dashed) components; measurements have been taken for $\theta=\left[-90^{\circ},+90^{\circ}\right]$ and $\phi=\left[0^{\circ}\right.$ (black $),+45^{\circ}($ red $), 90^{\circ}($ green $),-45^{\circ}($ blue $\left.)\right]$. Center and Right: $30 \mathrm{GHz}$ simulated feed horn pattern, co- (center) and cross-polar (right) components; the feed data consist of 72 cuts with $\theta=\left[0^{\circ}, 180^{\circ}\right]$ and $\phi=\left[0^{\circ}, 5^{\circ}, 10^{\circ}, \ldots, 355^{\circ}\right.$. Here we show four cuts for $\phi=\left[0^{\circ}(\right.$ black $),+45^{\circ}($ red $), 90^{\circ}$ (green $),-45^{\circ}($ blue $\left.)\right]$. In this case curves at $\pm 45^{\circ}$ are coincident.

\begin{tabular}{c|c|c}
\hline \hline 30 GHz Main Beam & Simulated Feed & Measured Feed \\
Max co & 51.001 & 50.93 \\
Max cx & 22.54 & 22.43 \\
XPD & 28.46 & 28.85 \\
\% DEP & 0.43 & 0.43 \\
FWHM X & 28.56 & 28.85 \\
FWHM Y & 36.39 & 36.39 \\
FWHM AVE & 32.47 & 32.63 \\
e & 1.27 & 1.26 \\
\hline
\end{tabular}

Table 1: Characteristics of the $30 \mathrm{GHz}$ Main Beam with Simulated and Measured feed horn as input: Maximum absolute value of the co and cross-polar components ( $\mathrm{dBi}$ ), cross-polar discrimination factor (XPD) (dB), Depolarization parameter (\% DEP), Full Width Half Maximum in X and Y direction calculated form a fit bivariate Gaussian contour (arcmin), Average Full Width Half Maximum (arcmin), Ellipticity (see [4] for definitions).

\section{3. $70 \mathrm{GHz}$ feed horns and main beam simulations}

The feed considered here is the FM n.86301. The measurements have been performed along the $\mathrm{E} / \mathrm{H} / \pm 45^{\circ}$ planes; all components (four co-polar and four cross-polar) have been measured with the "standard" horn as receiver. Moreover, the cross-polar component at $\pm 45^{\circ}$ have been tested with another FM LFI $70 \mathrm{GHz}$ horn (n.42805, "ideal" hereafter), with the aim of evaluate the contribution of the receiver feed horn introduced in the tested pattern. The complete measured pattern for the four planes is shown in Figure 2. Note that For the $\pm 45^{\circ}$ "standard" cross-polar component, the pattern is no longer cross-polar shaped and the comparison with the "ideal" curves suggest that a residual receiver component is actually measured. The cross-polar component in the $\mathrm{E}$ and $\mathrm{H}$ planes have been measured with the "standard" horn only: these two cuts do not reflect the real behaviour of a dual-profiled corrugated horn, that presents cross-polar components along principal planes almost null. The simulated feed horn pattern (not shown here) has a shape similar 
to the $30 \mathrm{GHz}$ case.

We performed main beam simulation for the $70 \mathrm{GHz}$ feed \#23 in the following cases: simulated feed horn (Figure 3 Top), measured feed horn with "ideal" at $\pm 45^{\circ}$ and "standard" cross-polar component in the $\mathrm{E}$ and $\mathrm{H}$ planes (Figure 3 Bottom), measured feed horn with "ideal" cross-polar component at $\pm 45^{\circ}$ and set to zero in the $\mathrm{E}$ and planes, measured feed horn with "standard" crosspolar component in the $\mathrm{E} / \mathrm{H} / \pm 45^{\circ}$ planes, measured feed horn with "standard" cross-polar at $\pm 45^{\circ}$ and set to zero in the $\mathrm{E}$ and $\mathrm{H}$ planes. For the co-polar components, no significant differences have been found in the main beam parameters. Comparing all the cases, the difference in the feed horn cross-polar component in the $\mathrm{E} / \mathrm{H}$ planes has a very low influence on the main beam shape and characteristics (see Table 2). In fact the telescope itself, due to the simmetry plane of the configuration, hardly modifies the original feed cross-polar components. Also setting to zero the "standard" cross polar components in the feed horn measured pattern in the $\mathrm{E} / \mathrm{H}$ planes does not make any significant difference, showing that the influence of the telescope is the dominant element.
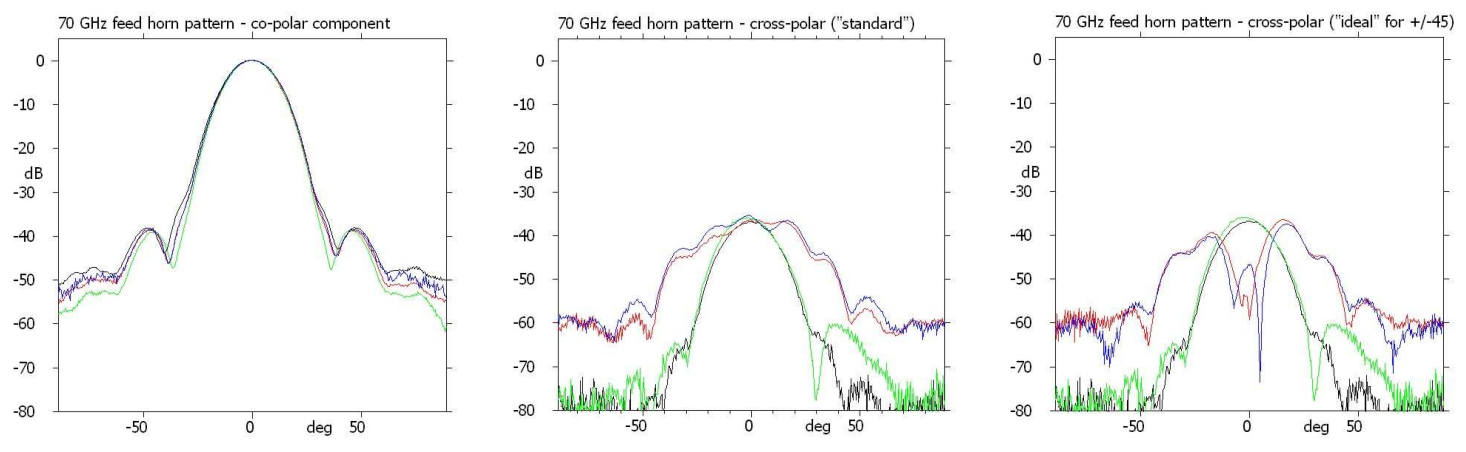

Figure 2: Left: co-polar cuts (E/H/ $\pm 45^{\circ}$ planes), Center: "standard" cross-polar cuts, Right: cross-polar cuts $\left( \pm 45^{\circ}\right.$ planes with "ideal" receiver, E/H planes with "standard" horn).

\begin{tabular}{c|c|c|c|c|c}
\hline \hline 70 GHz Main Beam & $\begin{array}{c}\text { Simulated } \\
\text { Feed }\end{array}$ & $\begin{array}{c}\text { Measured } \\
\text { Feed }\end{array}$ & $\begin{array}{c}\text { Meas. Feed } \\
\text { standard cx } \pm 45^{\circ}\end{array}$ & $\begin{array}{c}\text { Meas. Feed } \\
\text { null cx } 0^{\circ} / 90^{\circ}\end{array}$ & $\begin{array}{c}\text { Meas. Feed } \\
\text { standard } \pm 45^{\circ} \\
\text { null cx } 0^{\circ} / 90^{\circ}\end{array}$ \\
Max co & 58.802 & 58.695 & 58.698 & 58.698 & 58.701 \\
Max cx & 30.791 & 30.592 & 31.002 & 30.545 & 30.811 \\
XPD & 28.011 & 28.104 & 27.695 & 28.153 & 27.889 \\
\% DEP & 0.376 & 0.439 & 0.435 & 0.421 & 0.421 \\
FWHM X & 11.641 & 11.751 & 11.759 & 11.749 & 11.758 \\
FWHM Y & 14.595 & 14.624 & 14.606 & 14.618 & 14.560 \\
FWHM AVE & 13.117 & 13.188 & 13.182 & 13.184 & 13.179 \\
e & 1.254 & 1.244 & 1.242 & 1.244 & 1.242 \\
\hline
\end{tabular}

Table 2: Characteristics of the $70 \mathrm{GHz}$ Main Beam (See Table 1 for parameters definition and Section 3 for cases description). 

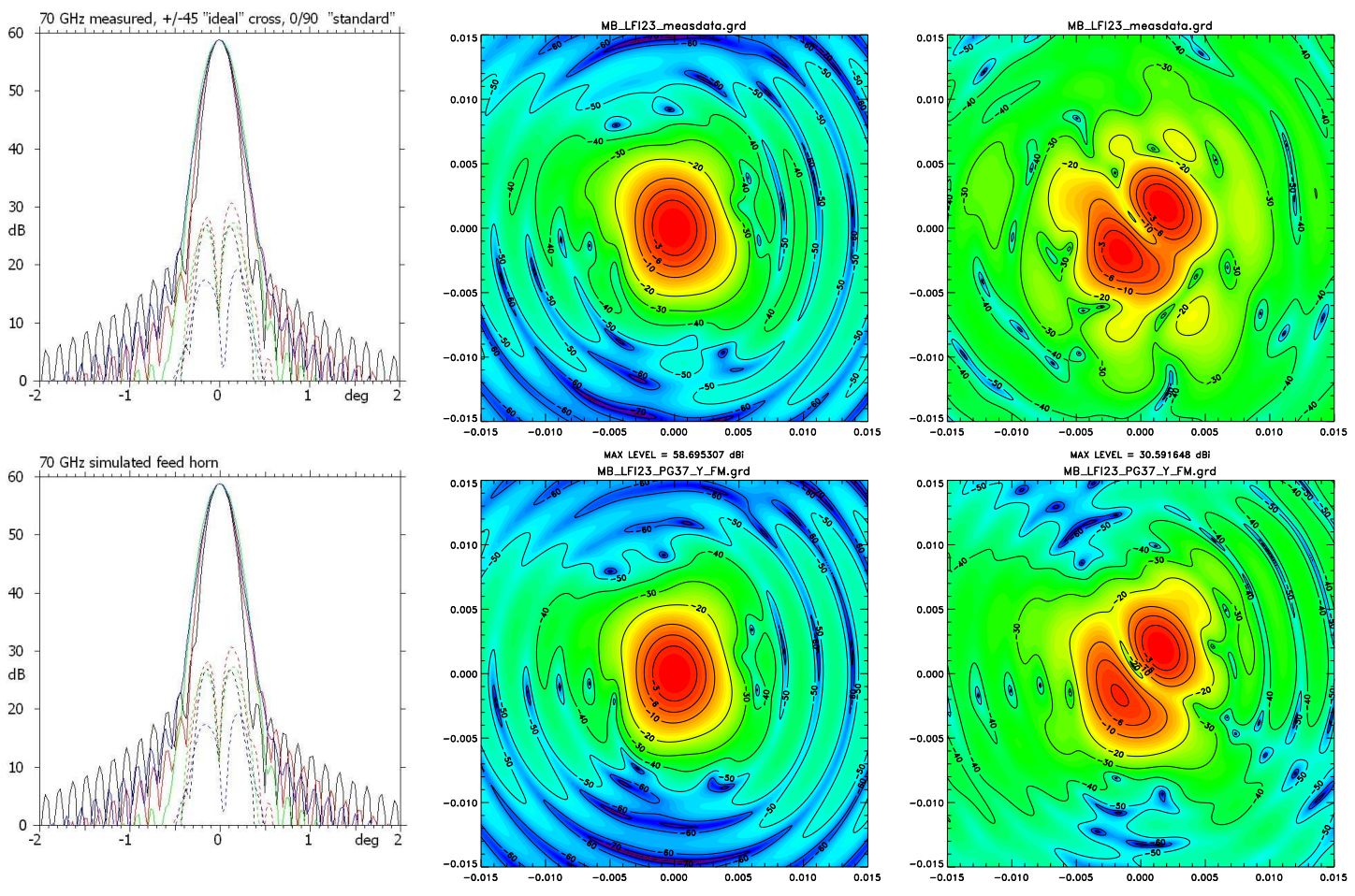

Figure 3: Top: measured feed horn case, Bottom: simulated feed horn case. Left Main beam co- and cross-polar cuts, calculated on the $\mathrm{E} / \mathrm{H} / \pm 45^{\circ}$ planes. Center and Right: Main beam co- and cross-polar components in $\mathrm{UV}$ grid. $\mathrm{U}$ and $\mathrm{V}$ range from -0.026 to $0.026\left(\theta \leq 1.5^{\circ}\right)$. The colour scale goes from -90 to $0 \mathrm{~dB}$. The lines in the contour plots, normalized at the power peak, are $-3,-6,-10,-20,-30,-40 \mathrm{~dB}$.

\section{Perspectives}

Future activities will regard both measurements and simulations. In particular we plan to perform: pattern measurements of more LFI FM feed horns at 30 and $70 \mathrm{GHz}$ with "ideal" receiver and related simulations, pattern measurements of $44 \mathrm{GHz}$ LFI FM feed horns with "ideal" receiver and related simulations, measurements of cross polar components in the $\mathrm{E} / \mathrm{H}$ planes with "ideal" receiver, evaluation of induced systematic effects and impact on scientific data.

\section{References}

[1] O. D’Arcangelo, P. Battaglia, M. Bersanelli, L. Figini, G. Mari, R. Nesti, M. Pecora, M. Sandri, A. Simonetto, C. Sozzi, F. Villa, Performance of the Planck-LFI feed horns, Qualification Model Proc. of 28th ESA Antenna Workshop on Space Antenna Systems and Technologies (Noordwijk, The Netherland, June 2005), WPP 247, Part 2, pp 965-971, 2005.

[2] K. Pontoppidan, Technical description of GRASP9, TICRA, 2005.

[3] A.C. Ludwig, The definition of cross polarization, IEEE Transactions on Antennas and Propagation, 1973, 21:116-119.

[4] M. Sandri, Planck-LFI: optical performances and systematic effects, PhD Thesis, 2005.

[5] R. Coccioli, G. Pelosi, R. Ravanelli, Combined mode matching integral equation for feeders optimization, 1996. 